\title{
Analysis of the electromagnetic radiation generated by a multipactor discharge occurring within a microwave passive component
}

\author{
M. Jiménez ${ }^{1}$, B. Gimeno ${ }^{2}$, C. Miquel-Espanya ${ }^{3}$, D. Raboso ${ }^{3}, \mathrm{~S}$.

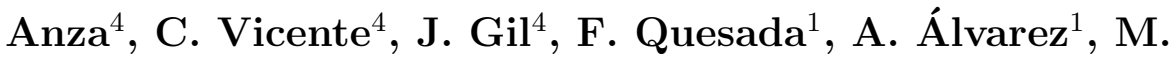 \\ Taroncher $^{5}$, M. Reglero ${ }^{5}$ and V. E. Boria ${ }^{5}$ \\ 1 Universidad Politécnica de Cartagena, Murcia, Spain \\ 2 Departamento de Física Aplicada y Electromagnetismo - ICMUV, Universidad de \\ Valencia, Spain \\ 3 European Space Agency (ESA), European Space Research and Technology Center \\ (ESTEC), Noordwijk, The Netherlands \\ 4 Aurora Software and Testing S.L., Valencia, Spain \\ 5 Departamento de Comunicaciones - ITEAM, Universidad Politécnica de Valencia, \\ Spain \\ E-mail: benito.gimeno@uv.es
}

\begin{abstract}
Multipactoring is a non-linear phenomenon that appears in highpower microwave equipments operating under vacuum conditions and causes several undesirable effects. In this manuscript, a theoretical and experimental study of the RF spectrum radiated by a multipactor discharge, occurring within a realistic microwave component based on rectangular waveguides, is reported. The electromagnetic coupling of a multipactor current to the fundamental propagative mode of a uniform waveguide has been analyzed in the context of the microwave network theory. The discharge produced under a single-carrier $\mathrm{RF}$ voltage regime has been approached as a shunt current source exciting such a mode in a transmission-line gap-region. By means of a simple equivalent circuit, this model allows predicting the harmonics generated by the discharge occurring in a realistic passive waveguide component. Power spectrum radiated by a third order multipactor discharge has been measured in an E-plane silver-plated waveguide transformer, thus validating qualitatively the presented theory to simulate the noise generated by a single-carrier multipactor discharge.
\end{abstract}

PACS numbers: 84.40.Ua, 41.20.Jb, 29.27.-a, 52.20.Fs 


\section{Introduction}

Multipactor effect is a non-linear resonant phenomenon typically observed in high-power microwave subsystems such as RF satellite payloads and particle accelerator structures. It is often considered undesirable, since it can load the cavity [1], dissipate power, increase the noise level [2], and cause heating of the walls and outgassing. Concerning multipactor in particle accelerators, two types of effects can be distinguished: $\mathrm{RF}$ multipactoring in the structures [3, 4], and beam-induced multipactor process driven by the field of the bunched beam [5, 6]. Multipactor effect has been deeply investigated in several types of microwave waveguides with simple geometries, such as the parallelplate guide [7, 8], the rectangular waveguide [10] and the coaxial transmission-line $[11,12,13,14]$. However, the interaction of a multipactor discharge occurring within a realistic waveguide component has not been deeply studied so far.

In this work, our aim is to study the propagation of the electromagnetic energy radiated by a multipactor discharge inside a passive microwave waveguide component. First, we have shown that the radiation of a multipactor current, occurring within a uniform waveguide, can be approached as the problem of a time-harmonic current density exciting a waveguide. Secondly, such current has been modelled, following a simple network theory, by means of a shunt current generator loading the waveguide section where the discharge takes place. Next, a single-mode transmission-line equivalent circuit for the analysis of a rectangular waveguide component is proposed. The timeharmonic sources of this circuit are a voltage and a current generators accounting for the RF power supply and for the discharge effect, respectively. Thus, in this model, the radiated energy by the event is coupled to the microwave structure. In addition, following this procedure, the non-linear feature of the single-carrier multipactor effect is taken into account. In order to validate the proposed model, an experiment based on a silver-plated WR-75 E-plane rectangular waveguide transformer has been carried out. The set-up required for this experiment, as well as the calibration technique employed to measure data, are both detailed. The power spectrum of the fields radiated by a thirdorder multipactor discharge has been detected (for the first time to the knowledge of the authors), and it has been compared with the theoretical results provided by this model, thus demonstrating the capability of the new proposed theory to predict qualitatively the distortion generated by a multipactor event.

\section{Radiation of a multipactor discharge occurring in a rectangular waveguide}

From the classical microwave theory, it is well known that passive microwave components, such as filters, diplexers and multiplexers, can be analyzed in terms of transmission-line circuits driven by time-harmonic sources. Thus, microwave network analysis transforms the vector electromagnetic field equations into multimode equivalent circuit models based on transmission-lines [15]. In this letter, we have focused on the 
analysis of an E-plane rectangular waveguide transformer, but the developed theory is completely general. In this scenario, the propagation of the fundamental $T E_{10}$ mode in such passive waveguide component can be described by means of the circuit shown in Fig. (1), omitting the current source. In this network, a series of transmission lines (representing the waveguide sections of the component) of wave impedance $Z_{0}$ are connected through capacitive junctions (represented by rectangular boxes) placed between adjacent guides. The circuit is excited by a single-carrier time-harmonic RF voltage source of frequency $f=\omega /(2 \pi)$ and amplitude $V_{R F}$. A time dependence $e^{+j \omega t}$ is assumed throughout this work, $j=\sqrt{-1}$ being the imaginary unit.

In the context of the microwave network theory, the presence of a transverse electric wire-current radiating within an infinite and uniform cross-section waveguide can be modelled in terms of a time-harmonic current source. Following [15], the telegrapher equations of this waveguide section containing such wire-current have to be modified according to,

$$
\begin{aligned}
& \frac{\mathrm{d} V(z)}{\mathrm{d} z}=-j \beta_{0} Z_{0} I(z) \\
& \frac{\mathrm{d} I(z)}{\mathrm{d} z}=-j \frac{\beta_{0}}{Z_{0}} V(z)-I_{m} \delta\left(z-z^{\prime}\right)
\end{aligned}
$$

where $z$ is the longitudinal direction of the line, $V(z)$ and $I(z)$ are the equivalent circuit voltage and current associated to the fundamental propagative mode, respectively, and $\beta_{0}$ is the propagation constant. Note that for our case the wave impedance is given by $Z_{0}=\omega \mu_{0} / \beta_{0}$. The last term in $(1 b)$ refers to the insertion of the electric transverse wire-current at the point $z=z^{\prime}$, which is acting as a shunt current source of amplitude $I_{m}$. In this equation, the Dirac delta function ensures that this current is located only at the point $z=z^{\prime}$.

The classical multipactor theory for a parallel-plate waveguide region $[7,8,9]$ gives correct predictions in real rectangular microwave structures when the propagating mode is the fundamental $T E_{10}$ mode, and when the gap is very small compared with the rest of dimensions [10]. This is the case for the central section of the E-plane waveguide transformer analyzed in this work (see Fig. 2(a), Fig. 2(b) and Table I), where the gap (oriented in the $y$ direction) is approximately 60 and 125 times smaller than the $x$ and

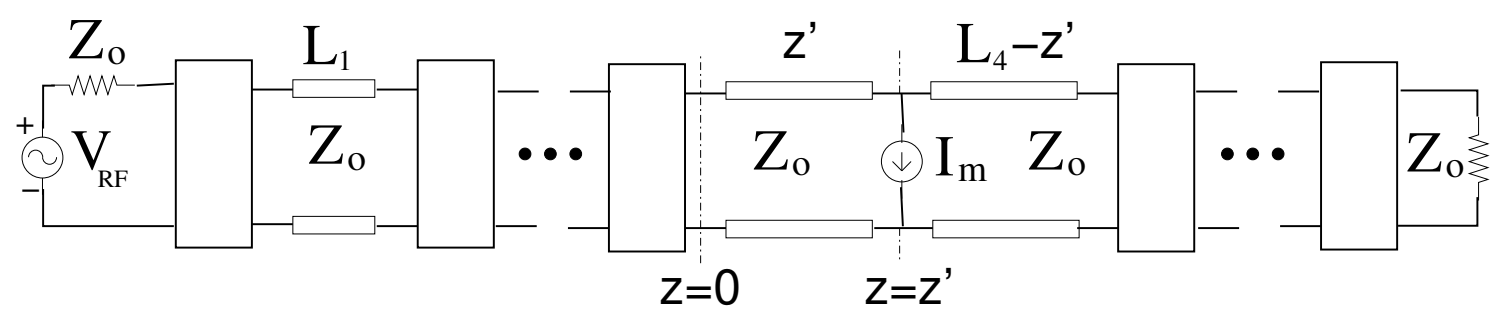

Figure 1. Circuit model of the considered E-plane waveguide transformer. The wave impedance of the fundamental rectangular mode has been denoted as $Z_{0}$. The voltage amplitude of the RF voltage generator is $V_{R F}$. The equivalent multipactor current sources are represented by $I_{m}$. 
$z$ dimensions, respectively. As a consequence, such classical model has been used in this work for the description of a double-surface multipactor discharge $[2,16]$.

A first simple model to describe the double-surface multipactor effect is based on a unique effective electron which accumulates the total charge and mass of the generated electrons. Thus, the charge accumulated in the discharge can be expressed as $-e n_{e}$, where $n_{e}$ is the number of elementary charges and $-e$ is the electron charge. This effective electron is driven by a time-harmonic single-carrier RF voltage of frequency $f$. For the Sombrin's (or Vaughan's) approach, a constant initial velocity for the secondary emitted electrons is assumed $[8,9]$. In order to obtain an analytical description of the phenomenon, Coulombian repulsion of the electron cloud is neglected in this model. Thus, the direct integration of the non-relativistic Lorentz force allows to describe the electron dynamics and to derive the multipactor resonance conditions (which can be expressed in terms of analytical forms, as detailed in [8, 9]). Of course, this model follows the classical theory of multipactor, with the limitations that the spread of the electron initial velocity as well as the Coulombian repulsion have not been considered. However, it is applicable for qualitative consideration and constitutes a first step in this theory that can be completed in further works.

In this model, the external field experienced by the effective electron is thus provided by the $y$-component of the RF single-carrier electric field $\left(E_{y}\right)$. At the initial stage of the discharge, the radiated electromagnetic field emitted by this electron is neglected in the equation of motion because it is very low in comparison with the RF single-carrier field (see $[7,8,9,10,11,12,13,14]$ ).

Following with the derivation of the problem and employing the above model, an equivalent $y$-oriented uniform multipactor wire-current of length $d$ is defined and expanded in terms of an infinite Fourier series with amplitudes $i_{m}$ with harmonic index $m=1,3,5 \ldots$, as shown in equation (5) of [2]. Each of these odd harmonics is oscillating at a frequency given by $f_{m}=m(f / N), N$ being the multipactor order. Thus, the inherent non-linearity behavior of the multipactor effect is clearly evidenced. In the present formulation, we assume that these wire-currents $i_{m}$ are radiating within a rectangular waveguide of dimensions $a$ and $b$. This set of time-harmonic currents can be represented by the following electric current singular densities:

$$
\vec{J}_{m}(x, y, z)=n_{e} i_{m} \delta\left(x-\frac{a}{2}\right) \delta\left(z-z^{\prime}\right) \hat{y} ; m=1,3,5 \ldots
$$

Obviously $d=b$ for these elemental wire currents.

Following the formulation developed in [15], the next step is to expand these multipactor current densities in terms of the electric fields of the TE and TM modes, resulting in

$$
\vec{J}_{m}(x, y, z)=\sum_{p} I_{m_{p}}(z) \vec{e}_{p}(x, y)
$$

where $I_{m_{p}}$ are the expansion coefficients, and $\vec{e}_{p}$ are the transverse electric vector-mode functions, which are normalized according to

$$
\int_{C S} \vec{e}_{p} \cdot \vec{e}_{q} d S=\delta_{p, q}
$$


where $C S$ represents the rectangular waveguide cross-section, and $\delta_{p, q}$ is the Kronecker delta. Equation (3) indicates that each $i_{m}$ current might excite the full set of TE and TM waveguide modes at different $f_{m}$ frequencies. However, in this first approach of the problem, we will only consider the first $(p=1)$ fundamental $T E_{10}$ mode, which is represented by the coefficient $I_{m_{1}} \equiv I_{m}$. Finally, the amplitudes of the equivalent multipactor current sources are easily obtained using the orthonormalization properties of the vector-mode functions (4):

$$
I_{m}=\sqrt{\frac{2 b}{a}} n_{e} i_{m}
$$

\section{Application to the multipactor radiation analysis of an E-plane waveguide transformer}

An E-plane silver-plated rectangular waveguide transformer, which is shown in Fig. 2(a), has been used to verify the proposed theory. Its geometrical dimensions are summarized in Fig. 2(b) and Table 1 (note that $a=19.05 \mathrm{~mm}$ for all waveguides). The electrical response of this device has been computed in a wide frequency band with an efficient full-wave modal software tool called FEST3D $\ddagger$. In this code, the analysis of a planar waveguide junction is performed by means of the numerical solution of a first kind Fredholm integral equation. The unknown of the problem is the tangential electric field, which is decomposed as a linear combination of basis functions; the formulation directly leads to a representation of the problem in terms of a generalized impedance matrix (see details in $[17,18]$ ), which allows an efficient characterization of the capacitive steps of the waveguide transformer. For the solution of the resulting integral equation we have considered 30 accessible modes (including both propagative and evanescent modes), 600 localized modes (evanescent modes), and 30 basis functions. The simulated and measured scattering parameters are shown in Fig. 2(c), where a good agreement is observed in the single-mode frequency band of the WR-75 waveguide; experiments out of the single mode band have not been performed because of its difficulty in the calibration of the instruments.

In order to study the interaction between a multipactor discharge and the waveguide transformer, the single-mode circuit shown in Fig. (1) has been analyzed in terms of impedance matrices. The rectangular boxes representing the E-plane steps have been electromagnetically characterized by means of the aforementioned full-wave modal software FEST3D. Finally, the Kirchhoff's laws have just been applied to evaluate the transmitted and reflected power considering the presence of the voltage and current sources. The voltage generator provides the RF supply to the circuit, which has been properly related to the input power $P_{\text {inp }}$; its amplitude and frequency are $V_{R F}$ and $f$, respectively. On the other hand, the amplitudes and the frequencies of the harmonic current sources are given by $I_{m}$ and $f_{m}$, respectively.

$\ddagger$ http://www.fest3d.com 
Table 1. Dimensions of the E-plane waveguide transformer.

\begin{tabular}{ccc}
\hline WG Section & $L_{i}(\mathrm{~mm})$ & $b_{i}(\mathrm{~mm})$ \\
\hline in & 20.00 & 9.53 \\
1 & 8.11 & 5.46 \\
2 & 8.03 & 1.44 \\
3 & 8.24 & 0.44 \\
4 & 40.04 & 0.32 \\
5 & 8.24 & 0.44 \\
6 & 8.03 & 1.44 \\
7 & 8.11 & 5.46 \\
out & 20.00 & 9.53 \\
\hline
\end{tabular}

The differences in the frequency response between the single-mode circuit model and the numerical results provided by the software FEST3D are plotted in Fig. 2(d): a very good agreement in the single-mode operation band of the WR-75 waveguide (10$15 \mathrm{GHz}$ ) is observed, whereas this tendency is degraded in the out-of-band frequency region because of the excitation in the discontinuities of the higher-order $T E_{1 n}$ and $T M_{1 n}$ modes (note that $n$ is even due to the symmetry existing in the complete structure, i.e. $n=0,2,4,6 \ldots)$.

The discharge current has been located at the point of maximum field within the central section of the waveguide transformer, which has been found by plotting the $y$ component of the RF electric field magnitude $\left(E_{y}\right)$ as a function of the axial coordinate for an input RF power of $P_{i n p}=1 \mathrm{~W}$, as depicted in Fig. 3. Results obtained with the transmission line model and the FEST3D code are successfully compared, demonstrating that the effect of the higher-order modes generated in the adjacent discontinuities of the central waveguide is negligible in comparison with the fundamental propagative $T E_{10}$ mode contribution. A standing wave pattern is formed by the interference between the incident and reflected waves. For the frequency selected $f=12.466 \mathrm{GHz}$, the maximum field arises in the point $z^{\prime}=14.7 \mathrm{~mm}$.

At this point, and following the classical double-surface multipactor theory $[7,8]$, we define an equivalent voltage $V$ as the line integral of the electric field between the two points on the surfaces of the structure. In this case, since the propagating mode is the fundamental one, there is no field variation along $y$ axis. Therefore, the voltage between any point $(x, z)$ along the top and bottom surfaces (constant $y$ planes) is simply the multiplication of the electric field at that point by the gap distance. The authors have calculated the maximum electric field $\left(E_{y_{1 W}}\right)$ of the central section of the transformer assuming an input power of $P_{i n p}=1 \mathrm{~W}$ (shown in Fig. 3) resulting in $E_{y_{1 W}}=18581.5$ $\mathrm{V} / \mathrm{m}$. Finally, for any RF input power $P_{i n p}$ the applied voltage in the critical gap region is simply calculated as $V=V_{1 W} \sqrt{P_{i n p}}$, where $V_{1 W}=E_{y_{1 W}} d$ and $\mathrm{d}$ is the gap distance $\left(d=b_{4}=0.32 \mathrm{~mm}\right.$ in this case). 


\section{Description of the experimental set-up}

An experiment to validate the present theory was carried out at the European Space Agency ESA/ESTEC RF High-Power Laboratory; the experimental set-up has been described in Fig. (4), where the DUT (Device Under Test) is the aforementioned Eplane waveguide transformer.

Several multipactor detection methods were used in parallel: nulling of the forward/reverse power at carrier frequency $(f)$, third-harmonic detection, chamber pressure recording, and electron probe to monitor qualitatively the emitted secondary electrons (installed out of the DUT). Diagnostic tests performed in this laboratory have shown that having sufficient free electrons travelling inside the critical gap is a necessary condition in order to obtain reliable data on the discharge thresholds. An optical fiber connected to an ultraviolet mercury lamp $(254 \mathrm{~nm})$ was used to generate photo-electrons in the vicinity of the waveguide gap region in order to initiate the discharge. Both the optical fiber (blue connector) and the electron probe were inserted in the RF waveguide circuit (preceding the waveguide transformer) through two venting holes, as shown in Fig. (5). The pressure achieved inside the used vacuum chamber was around $3 \cdot 10^{-7}$ mbar.

The measurements were performed using spectrum analyzers for both the nulling system and the harmonics system. The spectrum analyzers are set up to be able to record (and retain on their screen) quick changes in the measurement. They were set up for the displaying of two traces, one trace was the live measurement and the second one was set to "Max Hold" so that fast spikes will be kept recorded in this trace. The central frequency in the spectrum analyzers was set to the relevant measured frequency (the carrier $f$ for the nulling system, and the different harmonics to be investigated in the harmonics generation, $\left.f_{m}=m(f / N)\right)$, and the frequency span was selected in the interval $100-200 \mathrm{KHz}$. The RF input power was increased in short steps until a discharge was triggered. The amplitude of the change in the nulling system is commonly used as an indicator of the severity/strength of the discharge. The measurements in the amplitude change of the harmonics system were performed for discharges of comparable strength according to the amplitudes found in the nulling system for these discharges.

The nulling system is set up superposing the input and the reflected signals, which are extracted from the experimental test-bed with two directional couplers. The sample of the input power is connected to a variable attenuator, and the reflected one is connected to a phase shifter device. Then the outcome of both signals is combined using a $3 \mathrm{~dB}$ hybrid, and the result is feed to a spectrum analyzer. During the tests the variable attenuator and the phase shifter are tuned to obtain a zero in the spectrum analyzer. This zero is very sensitive to changes in the amplitude/phase of the reflected signal, which makes it very appropriate to detect the multipactor discharges.

In the case of the higher order harmonics, the filtering was achieved by reducing the dimensions of the waveguide, filtering out the lower frequencies. The filtered signal of the different harmonics is properly amplified using LNAs (Low Noise Amplifiers) and 
feed to the spectrum analyzer.

The output section was calibrated using the following standard insertion-loss procedure. An RF cable was connected to the signal generator at one end, whereas the other end was connected to the spectrum analyzer. Then, the calibration frequency was set in the signal generator. The RF power was set to the highest level compatible with the rest of the setup, and the measured RF power at the end of the RF cable was recorded. The RF cable was disconnected from the spectrum analyzer and connected with an adapter to the WR-75 waveguide injecting the RF signal towards the output section. The spectrum analyzer was connected to measure the relevant harmonics RF path. Now, the RF power measured by the spectrum analyzer is also recorded. The difference between both measurements is the calibration factor for the used frequency. This procedure was repeated for the different frequencies to be measured. There was a first discharge at a certain power level, but after this first discharge a second discharge was found at a higher (RF power) level; all the other successive discharges were found in the vicinity with a dispersion of $5-10 \%$.

It should be remarked that the threshold input power and the amplitudes of the measured harmonics were reproducible with a relative error around $10 \%$. There are several explanations for this, such as the randomness of the electron's speed once they are emitted from the metal, the initial electron population inside the cavity, the degradation of the cavity surfaces, etc. Further research must be performed to clarify this point, which is common in all the experiments related with multipactor phenomenon.

\section{Results}

A multipactor discharge was detected in the central region of the waveguide transformer with an input peak power of $P_{i n p}=2400 \mathrm{~W}$ at $f=12.466 \mathrm{GHz}$. A pulsed signal was employed with a duty-cycle of $2 \%$ and a pulse-width of $20 \mu \mathrm{s}$. For this input power we have calculated with FEST3D the region where the electric field is maximum (see Fig. 3), which has associated an equivalent voltage amplitude of $V=291.3 \mathrm{~V}$. For a frequencygap product given by $f \cdot d=3.99 \mathrm{GHz} \mathrm{mm}$ (in this case $d=b_{4}=0.32 \mathrm{~mm}$ ), this amplitude corresponds (following the classical multipactor theory [7, 8] implemented in the code ECSS Multipactor Tool $\S$ ) with a multipactor order $N=3$. A spectrum analyzer was employed in order to detect the RF radiated spectrum. In order to validate qualitatively the presented formulation, experimental data are compared with theoretical results in Fig. (6); the resulting RF harmonics have been marked in this figure. For multipactor order $N=3$, the frequency carrier coincides with the peak $m=3\left(f_{3}=f\right)$. The peak at frequency $f_{1}=4.16 \mathrm{GHz}$ was not recorded because this frequency is below the cut-off frequency of the WR-75 guide, so it can not be propagated. The resonances $m=5$ and $m=7$ have been detected for the first time to the knowledge of the authors. In the case $m=9$ we find that $f_{9}=3 f$, so it coincides with the standard 
third-harmonic detection technique. Measurements performed at other frequencies only detected the noise floor level, which was around $-95 \mathrm{dBm}$ in this experiment.

In the single-mode circuit presented in Fig. 1, the RF voltage generator was easily evaluated by means of the well-known formula $V_{R F}=\sqrt{8 Z_{0} P_{\text {inp }}}=3053.81 \mathrm{~V}$. On the other hand, it should be emphasized that the experimental set-up used in this work does not allow to measure directly the electron population generated in a multipactor event $\left(n_{e}(t)\right)$. As a consequence, the electron population was estimated as $n_{e}=10^{8}$ electrons for a good fitting of the $m=7$ harmonic (the other harmonics $m=5$ and $m=9$ were not considered for this purpose because the transmission coefficient of the complete structure was very low at their corresponding emission frequencies, as it can be observed in Fig. 2(d)). Obviously, the concordance between measurements and theoretical results is perfect for this harmonic, as depicted in Fig. 6. It should be remarkable that the noise level associated with both $m=5$ and $m=7$ harmonics has been detected for the first time (to the knowledge of the authors) in a multipactor experimental set-up for telecommunication applications (the $m=9$ harmonic is typically observed in the thirdharmonic multipactor detection method). Other interesting aspect to be considered is the different trend of the radiated power spectrum between the parallel-plate model, which is continuously decreasing (as reported in $[2,16]$ ), and the results provided by the presented model, where the transmission coefficient of the DUT is directly included in the formulation. Finally, we want to remark that the discrepancies observed in Fig. 6 between theoretical and measured data for such harmonics are mainly attributed to the single-mode character of the developed model. Additionally, the presence of the optical fiber and the electron probe (preceding the DUT, as shown in Fig. 5) has not been considered in the electromagnetic characterization of the experiment, and obviously might disturb the obtained experimental results. An interesting future work would be to extend the presented formulation to a rigorous multimode network full-wave theory.

\section{Conclusions}

In conclusion, we have experimentally detected the $\mathrm{RF}$ spectrum radiated by a multipactor discharge occurring in the central gap-region of an E-plane silver-plated waveguide transformer implemented with rectangular waveguides. A formulation based on the classical microwave network theory has been derived to explain this experiment. In this context, the multipactor discharge is modelled with a shunt current source radiating at different frequencies, which excites the propagation of the TE and TM rectangular modes. Therefore, the inherent non-linear nature of the multipactor phenomenon is clearly evidenced with the presented circuit model. From a qualitatively point of view, comparison between measurements and theory validate the presented formulation. 


\section{Acknowledgments}

The Authors would like to thank ESA/ESTEC for having funded this research activity through the contracts "RF Breakdown in Multicarrier Systems" (Contract No. AO/1 4978/05/NL/GLC), and "Multipactor analysis in planar transmission lines" (Contract No. 20841/08/NL//GLC).

\section{References}

[1] R. Kishek and Y.Y. Lau 1995 Phys. Rev. Lett. 59 107-141

[2] E. Sorolla and S. Anza and B. Gimeno and A. M. Pérez and C. Vicente and J. Gil and F. J. Pérez-Soler and F. D. Quesada and A. Álvarez and V. E. Boria 2008 IEEE Trans. Electron Devices 552252

[3] C. Wang and K. Hsieh and L. H. Chang and M. C. Lin and K. R. Chu 2007 IEEE Trans. Applied Superconductivity 171285

[4] J.G. Power and W. Gai and S. H. Gold and A. K. Kinkead and R. Konecny and C. Jing and W. Liu and Z. Yusof 2004 Phys. Rev. Lett. 92164801

[5] F. Zimmermann 1997 A simulation study of electron cloud instability and beam induced multipacting in the LHC (CERN, Geneva, Switzerland, CERN-LHC-Project-Report-95)

[6] M. A. Furman and M. T. Pivi 2002 Physical Review Special Topics, Accelerator Beams 5124

[7] A. J. Hatch and H. B. Williams 1958 Phys. Rev. 112681

[8] J. R. M. Vaughan 1988 IEEE Trans. Electron Devices 351172

[9] J. Sombrin 1983 Technical Report (CNES, Toulouse, France)

[10] V. E. Semenov and E. I. Rakova and D. Anderson and M. Lisak and J. Puech 2007 Physics of Plasmas 14033501

[11] E. Somersalo and P. Ylä -Oijala and D. Proch and J. Sarvas 1998 Particle Accelerators 59 107-141

[12] R. Udiljak and D. Anderson and M. Lisak and V.E. Semenov and J. Puech 2007 Physics of Plasma 14033508

[13] V.E. Semenov and N. Zharova and R. Udiljak and D. Anderson and M. Lisak and J. Puech 2007 Physics of Plasma 14033509

[14] A. M. Pérez and C. Tienda and C. Vicente and S. Anza J. Gil and B. Gimeno and V. E. Boria and D. Raboso 2009 IEEE Trans. Plasma Science 372031

[15] L. B. Felsen and N. Marcuvitz 1994 Radiation and Scattering of Waves (IEEE Press Series on Electromagnetic Waves)

[16] B. Gimeno and E. Sorolla and S. Anza and C. Vicente and J. Gil and A. Pérez and V.E. Boria and F.J. Pérez-Soler and F. Quesada and A. Álvarez and D. Raboso 2009 Physical Review E 79 046604

[17] G. Gerini and M. Guglielmi and G. Lastoria 1998 IEEE MTT-Symposium Digest

[18] G. Conciauro and M. Guglielmi and R. Sorrentino 2000 Advanced Modal Analysis. CAD Techniques for Waveguide Components and Filters (John Wiley and Sons Ltd.) 

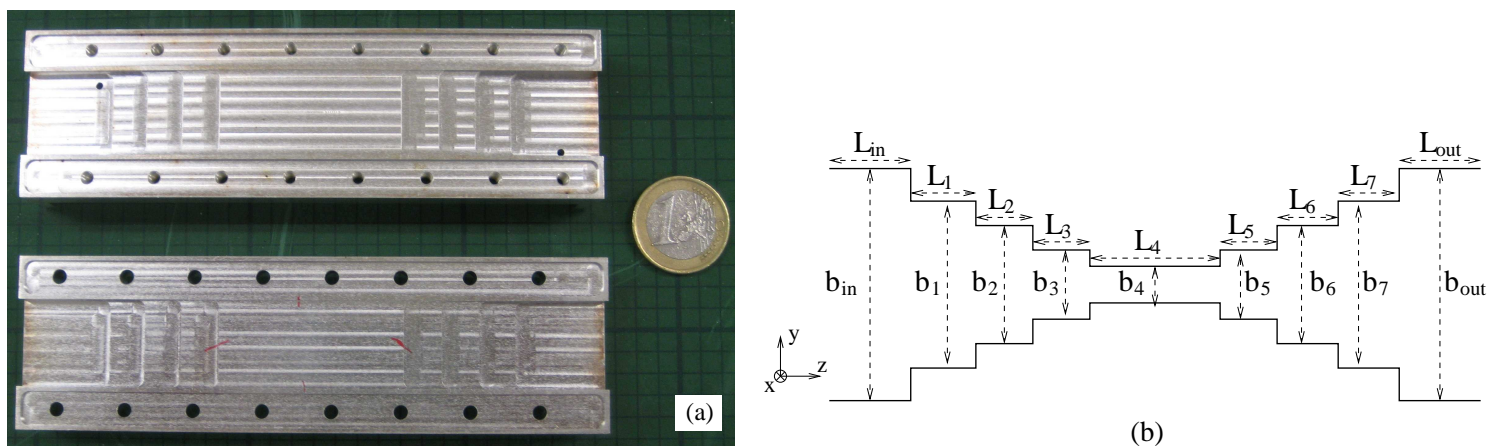

(b)
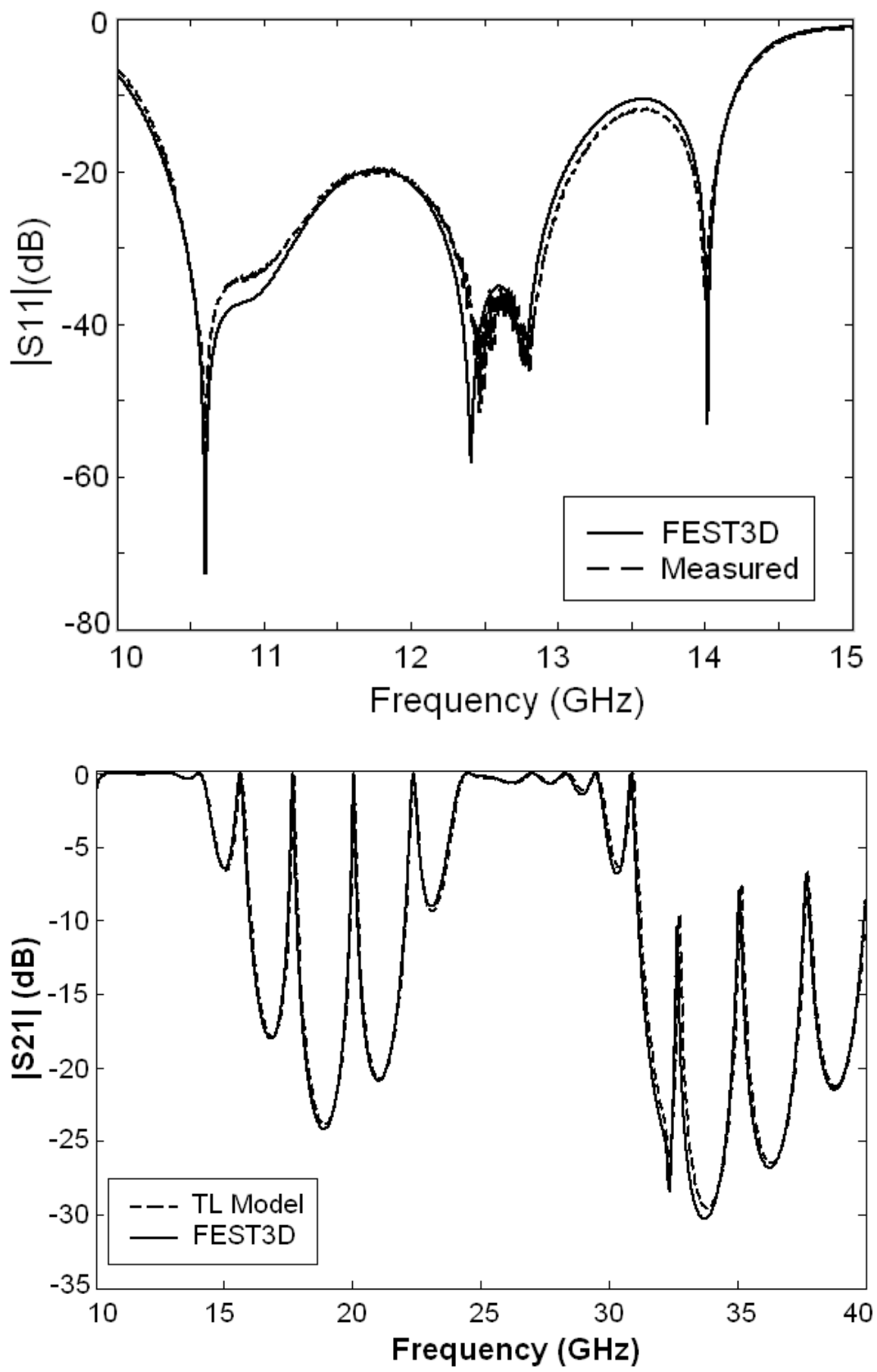

Figure 2. E-plane silver-plated WR-75 waveguide transformer. (a) Photo. (b) Drawing in the E-Plane. (c) Simulated (with FEST3D) and measured scattering parameters. (d) Comparison of the frequency response obtained with FEST3D and with the single-mode transmission line model. 


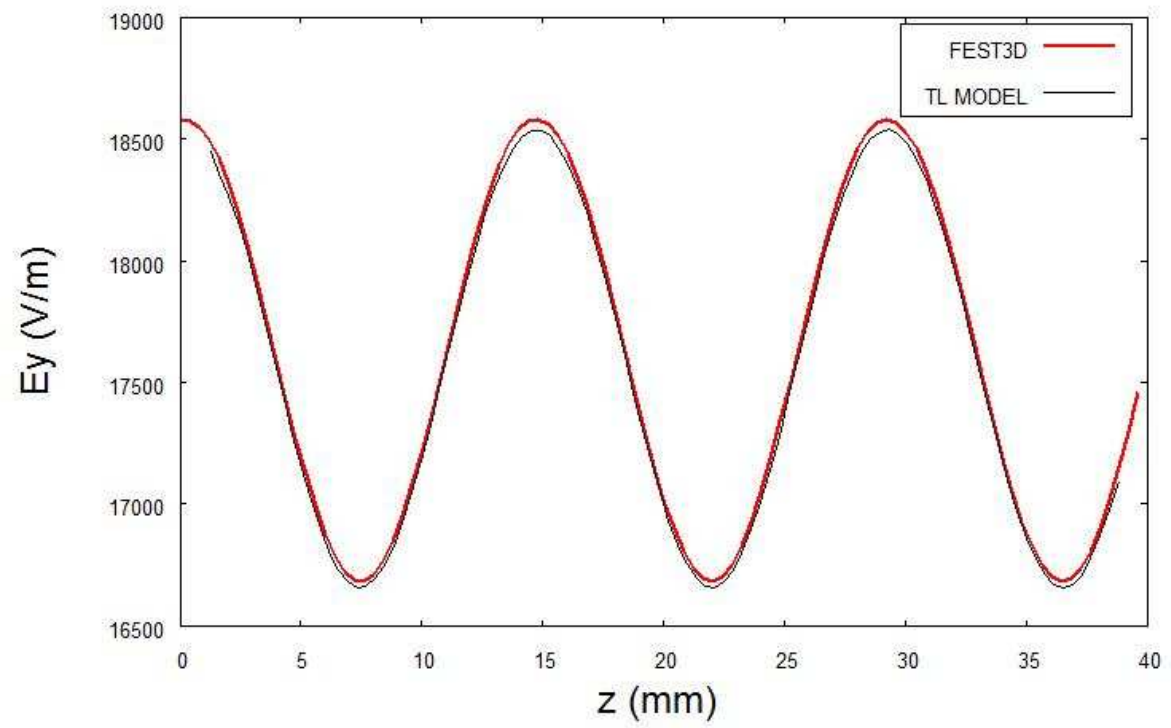

Figure 3. Magnitude of the $E_{y}$ field along the central section of the waveguide transformer. The input RF-power of the full structure is $P_{i n p}=1 \mathrm{~W}$, and the RF single-carrier frequency is $f=12.466 \mathrm{GHz}$.

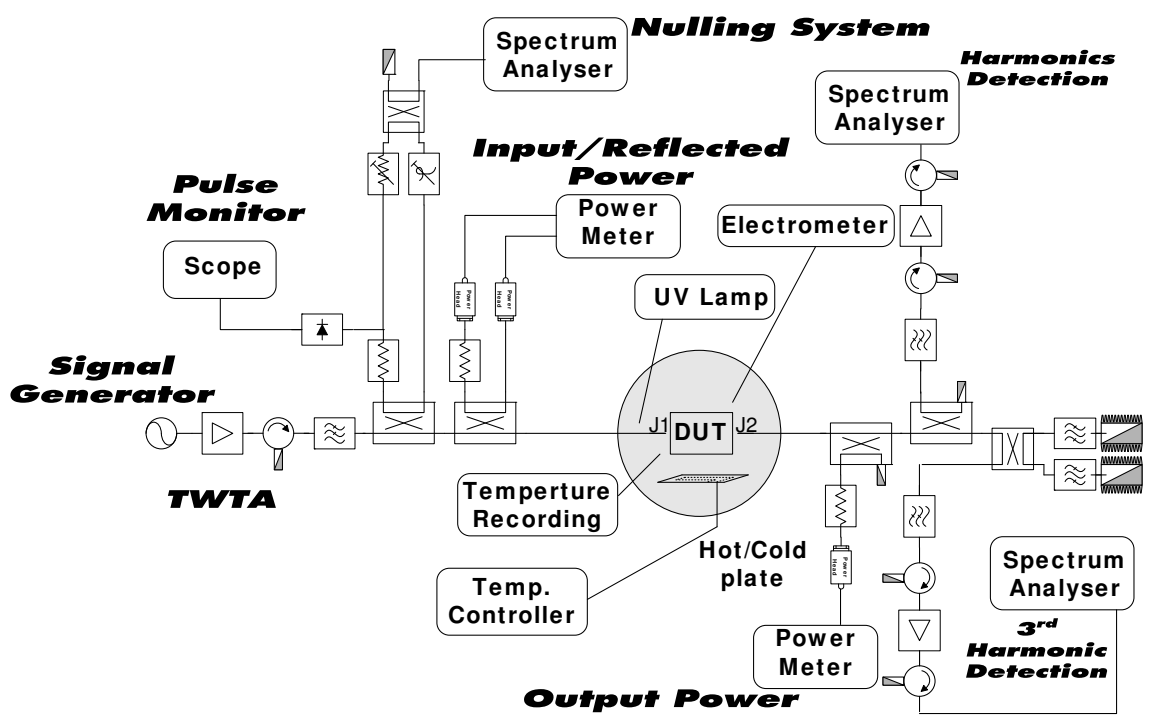

Figure 4. Test set-up used for the experiments performed at the ESA/ESTEC RF High-Power Laboratory. 


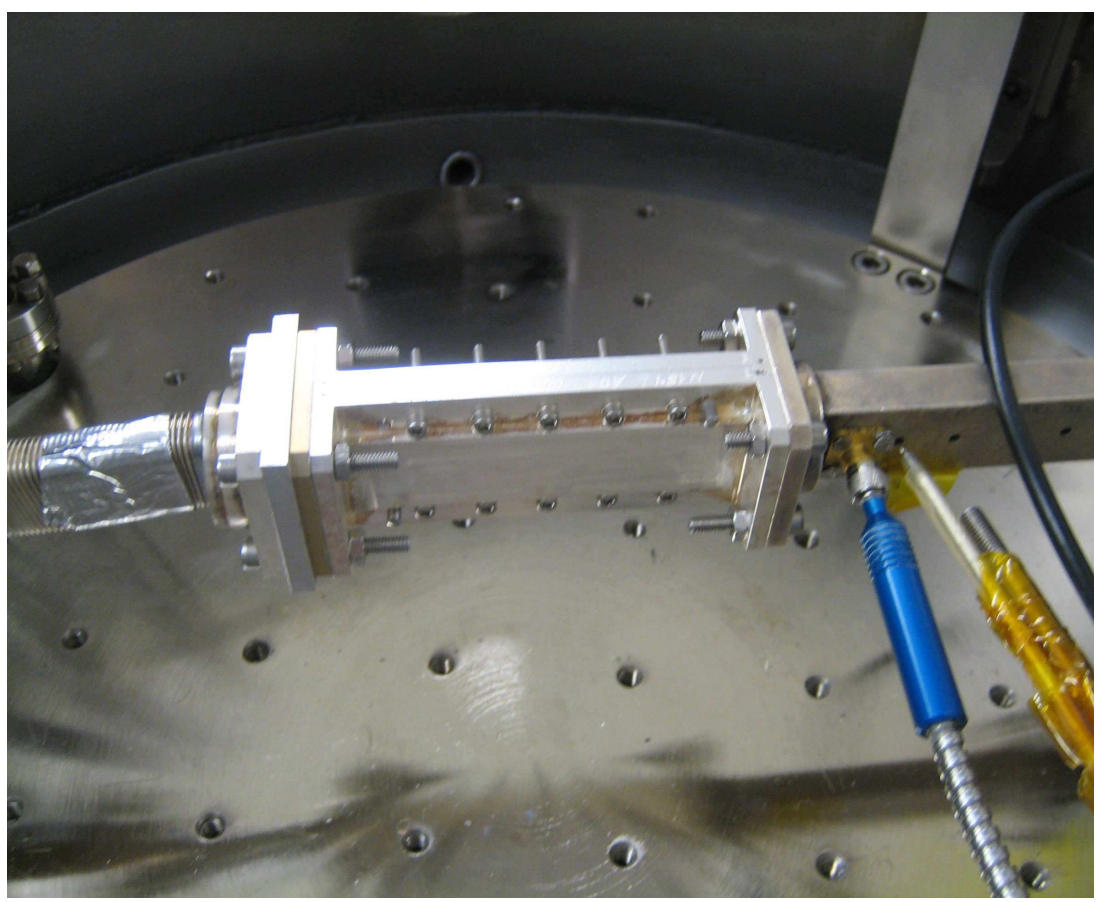

Figure 5. Photo of the E-plane waveguide transformer placed inside the vacuum chamber.

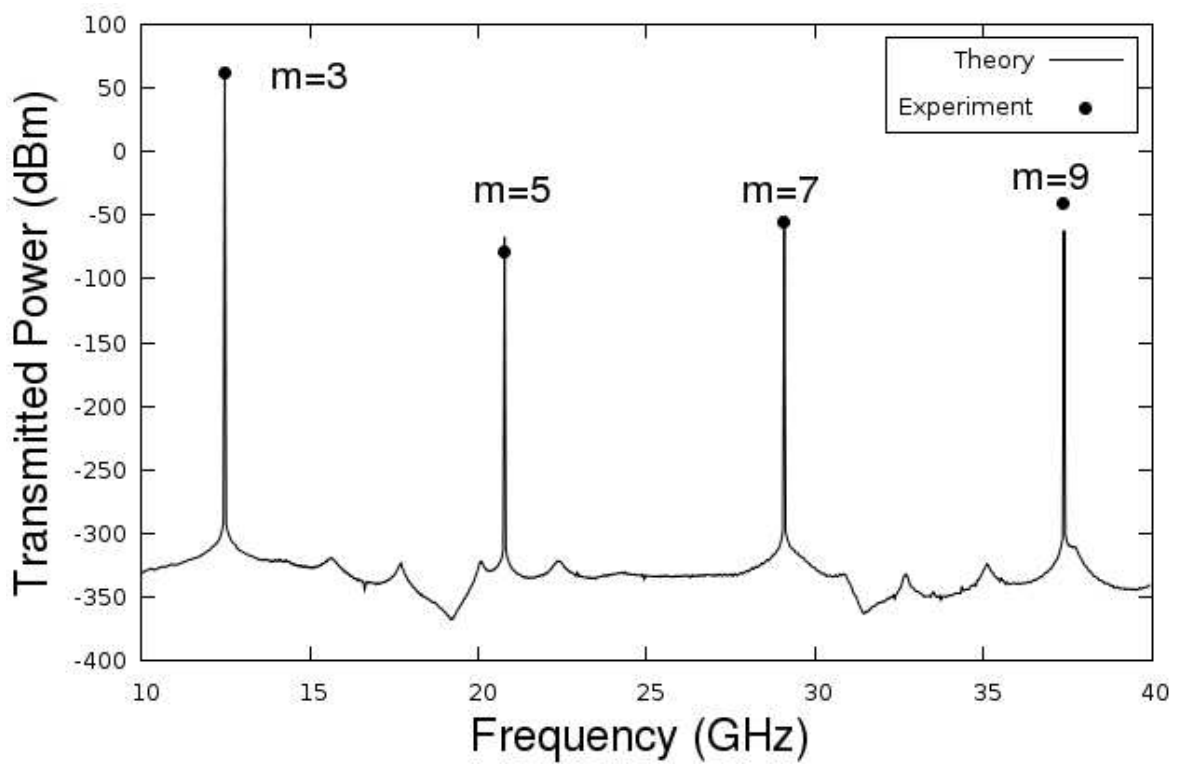

Figure 6. Transmitted power spectrum of the analyzed capacitive silver-plated waveguide transformer. 